\title{
La vivienda obrera del arquitecto D. Manuel María Smith Ibarra
}

\author{
ANa Julia GÓMEz GÓmez
}

RESUMEN

Manuel Maria Smith Ibarra (18791956) fue un arquitecto prolifero en construcciones para la burguesía vasca y considerado el introductor del estilo inglés en Bizkaia. Realizó también cinco proyectos de barrios obreros. Defendió la vivienda de baja densidad, en detrimento de la de pisos, considerándola la ideal para cualquier clase social.

\author{
ABSTRACT \\ Manuel María Smith lbarra (1879- \\ 1956) was an eclectic architect, built \\ for the baske bourgeoisie and the \\ introduced English Style in the Baske \\ Country. He also worked in low -class \\ housing, designed five working- \\ class quartes, and defending a low \\ density housing opposite to flat.
}

1. D. MANUEL MARIA SMITH IBARRA, UN ARQUITECTO PARA UNA BURGUESÍA

Manuel María Smith Ibarra nació en Bilbao en 1879 y estudió arquitectura en Madrid, concluyendo la carrera en 1903. Trabajó siempre en Bilbao y, aunque tuvo encargos en otras provincias, su obra se ubica en Vizcaya, principalmente en el municipio de Getxo, en los barrios de

* Tercer Ciclo. Departamento de Historia del Arte. UNED. 
Neguri, Las Arenas y Algorta. Fue uno de los arquitectos preferidos de la burguesía vasca, que le encargó los proyectos de sus residencias y chalets.

Arquitecto ecléctico, se expresaba con dominio en los estilos Montañés y Neovasco ${ }^{2}$, y además es considerado como el máximo introductor y representante del Estilo Inglés ${ }^{3}$ en el País Vasco, en especial de las variaciones del estilo Old English y del Reina Ana.

El grueso de la obra de Smith fue sobre todo residencial ${ }^{4}$ y su nombre se relaciona de inmediato a las grandes mansiones y chalets de Getxo y a los palacios ${ }^{5}$ como el de Artaza, construido para Víctor Chávarri, o

El Regionalismo Montañés creado por Leonardo Rucabado, fue una corriente arquitectónica que Smith supo variar produciendo un estilo Montañés propio, sobrio, personal e inconfundible, que tuvo una demanda importante en Bizkaia en las dos primeras décadas del siglo XX. Realizó obras de gran envergadura, como el palacio Lezama Leguizamón, el palacio del Marqués de Olaso,... para las cuales utiliza una magnifica silleria, con sobresalientes y elegantes torreones, y un repertorio decorativo recogido del gótico, renacimiento y barroco, siempre con mesura y sin recargamientos. En residencias más modestas se acerca más a lo popular, recreándose en solanas y miradores con armazón de madera, entramados de madera ficticios, línea de impostas sobresalientes, destacadas ménsulas, policromia y en conjunto una cierta rusticidad.

Los edificios neovascos parten de una fisonomía del caserio: sillarejo visto en los marcos de los vanos, ventanas, puertas, y en los esquinales; entramado ficticio;ladrillo visto en el frontón, a veces con imitación de huecos de ventilación triangulares; tejado a doble vertiente; caballete perpendicular a la fachada principal; cortafuegos decorativos; balcones con balaustres de madera o de hierro y chimeneas de ladrillo con copete...

3 La anglofilia vizcaína fue patente en diferentes ámbitos. La sociedad mostró su gusto por lo inglés en el deporte, en el vestir, en la proliferación de restaurantes y clubs y, por supuesto, en la arquitectura.

Smith interpretó a la perfección las diferentes variantes de ese estilo de arquitectura y le dotó de un carácter que arraigó rápidamente con el sentir de la sociedad vasca, retomando para el old English soluciones de las construcciones populares y adaptándolas a las necesidades del momento. Empleó torres de planta circular o cuadrada; asimetria general en la colocación de los elementos de la fachada;hastiales de remate triangular, utilización de teja plana; chimeneas decoradas con ladrillo o con revoco; varios tipos y tamaños de vanos en un mismo edificio; portalones de arco de medio punto; entramado ficticio, recto y también fue abundante el elemento curvo, definitorio del estilo. En cuanto al Reina Ana suprimió las torres, dejándolas sólo para las grandes mansiones. Evitó los entramados ficticios; utilizó el ladrillo visto en sardinel, espina de pez, creando composiciones romboidales, de dámero... En general, edificios más policromos y menos asimétricos que el Old English.

4 Egaña, Jaime. Prólogo del Catálogo Manuel María de Smith. Ed. Adarba, Madrid, 1933, pág. 5.

FullaONDO, Juan David. "Reestreno de un arquitecto" En Manuel Maria de Smith lbarra. Ed. COAM, Madrid, 1980, págs. 13-14.

Paliza Monduante, Maite. Manuel M. ${ }^{a}$ Smith Ibarra Arquitecto 1879 - 1956. Ed. Diputación Foral de Bizkaia, 1988.

5 El capítulo de palacios en la obra de Smith es interesante y amplio. Sólo en estilo montañés proyectó más de diez. El palacio de Víctor Chávarri, Marqués de Triano, considerada su obra más importante, la realizó en estilo inglés utilizando el Reina Ana. En base al mismo estilo inglés reformó el Palacio de Santa Clara, para José Maria de las Rivas. 
a los edificados para otros miembros de la alta burguesía vasca como Luis Lezama Leguizamón ${ }^{6}$, el Marqués de Olaso, o José Joaquín Ampuero.

Smith fue el arquitecto elegido por la oligarquía vasca, altamente enriquecida y que, en las tres primeras décadas de nuestro siglo, ve la necesidad de cambiar de residencia para mostrar su nuevo estatus social. Se trataba de que las nuevas residencias fueran acorde con el esplendor en el que se encontraban las clases altas, gracias al rápido enriquecimiento al que les había llevado la explotación minera y toda la infraestructura industrial que se había generado a su alrededor.

Smith supo responder a esta demanda, porque él mismo se sentía participe de este esplendor ${ }^{7}$, y ofreció las soluciones que el cliente demandaba.

\section{APLICACIÓN DE LAS IDEAS DE LA CIUDAD JARDÍN EN LOS PROYECTOS DE D. MANUEL MARIAA SMITH IBARRA PARA LA BURGUESIA VASCA}

Manuel María Smith acude, en 1907, al Congreso Internacional de Higiene y Demografía que se celebró en Berlín. En este congreso se debatió sobre la especulación de los terrenos en los pueblos, concluyendo al final de las jornadas con dos ideas principalmente, la primera que los Ayuntamientos se debían hacer cargo de reservar terreno a precio barato para construir y la segunda que era preferible la construcción de ciudades jardín.

Tan solo tres años después de su visita a Berlín viaja a Londres, al Congreso de Urbanismo en el que se estudió la Ciudad Jardín. Este viaje le permitió profundizar en los barrios obreros y en el planteamiento de la Garden City.

Para el estudio de los grandes palacios realizados por Smith, en estilo montañés e inglés ver: Paliza Monduante, Maite. Op. cit. págs. 262-285 y 112-145.

Sobre este palacio ver la publicación AC del GATEPAC, 1935, donde aparece una fotografía del palacio tachada, mostrándolo como ejemplo de lo que no debía hacerse en arquitectura. No deja de resultar un tanto sorprendente, porque para 1935 este tipo de edificios es algo puramente residual.

Ver también: FULLAONDO, Juan David. 1980: Op cit.

“Moradas señoriales. El Palacio Arriluce»En Revista Hermes. n. ${ }^{\circ}$ 3, Bilbao, 1917.

"Una residencia señorial en Neguri. (Vizcaya)" En. Revista de Arquitectura n. 127, Madrid, 1927, págs. 104- 109 .

Smith se relacionó por motivos de trabajo, amistad y parentesco con miembros de la alta burguesia vasca. 
La ciudad jardín y la calidad de la vivienda unifamiliar fueron para él objeto particular de reflexión a lo largo de su vida profesional, dejando opiniones escritas como la ponencia titulada "Urbanizaciones" que presentó en el Primer Congreso de Estudios Vascos, que se celebró en Oñate, en 1918.

Además de su preparación teórica, adquirida en congresos, conferencias y a través de la nutrida bibliografía que manejaba, Smith poseia cierta experiencia basada en la ciudad jardin. Había seguido de cerca la construcción de Neguri ${ }^{8}$, participando activamente en ella.

En 1911, Lucas Urquijo le encarga realizar una urbanización de casas para alquilar, Peñota, en Santurtzi, cuyos destinatarios iban a ser los ingenieros y altos cargos ingleses y alemanes que trabajaban en las factorías próximas ${ }^{9}$. El solar era un rectángulo entre Portugalete y Santurtzi, con una ligera inclinación hacia el mar. Smith lo divide por una calle central, a cuyos lados se dispone una hilera de casas adosadas a la izquierda y a la derecha dos casas bifamiliares y otras dos unifamiliares, todas diferentes.

En Peñota juega con los estilos Old English y el Reina Ana, con la asimetría que caracterizó sus proyectos, y con rica decoración en los hastiales a base de: entramados ficticios, balcones de madera, oriel windows, ventanas de cuarterones con sistema de abertura en guillotina...

Esta urbanización tenía un carácter inglés, al igual que Neguri o que Ondategui (1916) pero todas ellas distaban mucho de los planteamientos ingleses de la ciudad jardín. Había una similitud en cuanto a la estética, al cuidado por la higiene, la orientación, las zonas verdes comunitarias y los jardines individuales rodeando a cada casa, el espíritu de tranquilidad y la reminiscencia de casa de campo unida al confort de las nuevas técnicas y materiales, pero no trascendía de ello.

En 1912 trabaja en otro proyecto de las mismas características que Peñota, pero que no se llegó a realizar, se trata de la Urbanización Onchena ${ }^{10}$, en Bilbao. Este encargo de la familia Ibarra y Oriol, en unos terrenos entre las calles Sabino Arana y Manuel Allende, estaba destinado a clases medias. El proyecto era de casas unifamiliares, adosadas y con gran plasticidad, debido a la asimetria y variación en la decoración, primando el

8 El mismo año que Smith adquiere el título de arquitecto, 1904, comienzan las construcciones de los Chalets de Neguri, obra promovidas por la Sociedad de Terrenos de Neguri, cuyos promotores directos fueron: José Isaac Amann y Bulty, padre de su amigo Emiliano Amann y Amann; Daniel Aresti para el que realizó diferentes encargos y Valentín de Gorbeña.

s Ya existía un precedente de este tipo de construcción para extranjeros directivos de la empresa La Orconera, en Luchana.

to Archivo José María Smith Solaun: Rollo 318. Expediente. 18. 
estilo inglés. Planteó una urbanización con servicios comunitarios: farmacia, comercios, teléfono...

Por otro lado, en 1916, Sir Ramón de la Sota le encarga urbanizar una franja de terreno, prácticamente plano, entre las Arenas, Neguri, el paseo de Zugazarte y las vías del tren, lugar denominado con el nombre de Ondategui. Al igual que en Neguri, se trataba de una urbanización destinada a las clases altas, donde predominaron los chalets de tipo inglés y que conjugaron diferentes regionalismos, sobresaliendo el neovasco. Smith realizó el estudio de la urbanización calculando la construcción de 50 casas unifamiliares, pero a la hora de la construcción prefirió que participaran más firmas. El realizó las tres primeras casas, durante 1917, 1918 , 1919, y completaron el proyecto otros arquitectos, entre ellos Gregorio Ibarreche, Emiliano Amann, Ricardo Bastida...

Señalar definitivamente que su ideal se acercó siempre a la casa unifamiliar frente a la colectiva. La consideró como la preferible para el buen desarrollo de la institución familiar. Desde luego, era patente su conocimiento sobre este tipo de vivienda, más individualizada y de alto nivel, ya que fué uno de los arquitectos más prolíferos de su época pero su clientela no se encontraba entre los obreros. Los encargos para realizar vivienda obrera fueron muy puntuales y, excluyendo dos concursos públicos a los que se presentó, Calzadas y Solocoeche, los demás fueron realizados por mediación de los directivos de las empresas ${ }^{11}$, tanto en el caso de las Casas para empleados de Altos Hornos de Vizcaya, como para las Casas de la compañía Rica Hermanos.

\section{LAS VIVIENDAS OBRERAS DE D, MANUEL MARIA SMITH IBARRA}

A finales del siglo XIX las reivindicaciones obreras, canalizadas por la ideología socialista y manifestadas en importantes y repetidas huelgas generales, impulsaron a autoridades e industriales hacia la búsqueda de una solución al mal endémico de las penosas condiciones de vida y de vivienda que el proletariado venía arrastrando desde el comienzo de la industrialización.

\footnotetext{
"En el caso de Altos Hornos, Smith mantenia una relación laboral y de amistad con los directivos de esta empresa Victor Chávarri, Goyoaga, etc.

Con Víctor Chávarri tuvo una estrecha relación fuera del círculo de Altos Hornos, primero, en 1913 se le encargó el edificio de la Sociedad de Golf de Neguri y Chávarri supervisó directamente las obras, además meses después, en 1914, le presentó el proyecto para su residencia en Leioa, una de las obras cumbre de Smith: el palacio Artaza.
} 
En el País Vasco, se buscó en las Leyes de Casas Baratas, a partir de 1911 , el amparo del Estado para hacer menos onerosa la construcción de viviendas dignas para los menos favorecidos.

En Bizkaia las opciones generalizadas para afrontar la construcción fueron las de crear asociaciones y posteriormente Cooperativas. A nivel formal se optó mayoritariamente por la vivienda de baja densidad.

Aunque Manuel Maria de Smith demostró estar al corriente de la vivienda obrera que se realizaba en otros paises ${ }^{12}$, es indudable que no se dedicó a ella con demasiada devoción. Su amplia clientela de círculos burgueses llenaba su tiempo.

Por lo tanto, hablar de este arquitecto en relación con la vivienda para obreros no es habitual, ya que su nombre, como ha quedado señalado, va ligado de manera incuestionable a la plutocracia vasca, que vio en él el intérprete de sus deseos.

De todas maneras, en su brillante y prolifera carrera también hubo espacio para la vivienda de las clases menos pudientes, aunque en número escaso. Proyectó cinco barrios obreros, pero tan solo realizó dos, ambas en el municipio de Barakaldo: las casas baratas de Altos Hornos de Vizcaya en San Vicente y las casas para obreros de la fábrica Rica Hermanos, en el barrio de Irauregui. En todos los casos, fiel a la línea que marcó sus obras más onerosas, defendió la residencia de baja densidad en detrimento de la vivienda de pisos. y aplicó las mismas fórmulas arquitectónicas y estéticas que ennoblecían el resto de sus viviendas, dotándolas de calidad e individualidad.

Sin embargo, estas esporádicas incursiones de Manuel María Smith en la vivienda obrera pueden movernos a error, ya que no resulta fácilmente demostrable que fuese su interés personal en esta cuestión lo que le movió a comprometerse con este tipo de proyectos. Más bien lo hizo, inducido por el compromiso que tenia adquirido con conocidos, familiares o clientes, miembros de la alta burguesía vizcaína, que poseían factorias y reclamaban sus servicios a la hora de levantar las viviendas de sus obreros, o de las propias fábricas.

Sin embargo, su interés por los conceptos de calidad y confort de la vivienda le animaron a defender la casa de calidad para las clases trabajadoras como para las clases medias y a plasmar por escrito sus reflexiones. Lo hizo en diferentes memorias de proyectos, como es el caso del de Calzadas y el de Onchena.

Recordemos su asistencia a los congresos de Berlín y Londres señalados más arriba. 
Y por otro lado, es sobre todo en sus diseños más libres de viviendas para obreros donde muestra claramente su preferencia por la aplicación de las ideas de la Ciudad Jardín ${ }^{13}$, que ya guiaban sus obras más caras y cuidadas de Ondategui, Onchena y Peñota.

En el aspecto concreto de los proyectos de vivienda obrera que le ocuparon, señalar que a pesar de que las Casas de Altos Hornos se construian a través de la Sociedad de Casas Baratas de Baracaldo y Sestao, tras este nombre estaba la empresa ${ }^{14}$, que siguiendo las experiencias proteccionistas de otros paises europeos como Inglaterra, Francia, Alemania, Bélgica... consideró que ofreciendo viviendas a los obreros podía solucionar parte de los conflictos sociales que habían venido sucediendose en torno a la reivindicación de una vivienda digna. Era una manera de conseguir mano de obra no conflictiva y de sacar un provecho económico de los alquileres.

En el caso de los proyectos de Calzadas de Begoña y Solocoeche, se trataba de concursos públicos. El primero lo convocaba la Sociedad Anónima de Construcciones Baratas, formada por prestigiosos católicos de la villa bilbaína, con la finalidad de facilitar habitaciones económicas a los pobres, para salvaguardia de su moralidad y salud.

El presidente era el Conde Aresti, cliente de Smith desde 1904. De cualquier manera la invitación le llegó cursada a través la Asociación de Arquitectos, Smith se presentó al concurso junto con su compañero Arrupe, no quedando ganadores.

En el caso del Concurso de Casas para Solocoeche, se trataba de una convocatoria organizada por la administración pública, el ayuntamiento bilbaino que, a través del arquitecto municipal y concejal Tomás Bilbao, organiza un concurso público para realizar casa en altura, para la clase tra-

13 El concepto de ciudad jardin, que surge a mediados del siglo XIX, implica una nueva idea de ciudad autónoma y autosuficiente para un número determinado de habitantes, con unas condiciones de calidad de vida excelentes. Estas ideas provienen de Ebenezer Howard (1850-1928) que resume el pensamiento reformista anglosajón, con ciertos matices e influencias del socialismo fabiano inglès. Otro elemento importante es el del valor del terreno y su especulación. Howard se basa en el nuevo concepto de la plusvalía, dado por el americano Henry George, cuyos pensamientos se difunden por Europa y se apoyan en la idea de la tierra comunal para uso y disfrute de todos.

El elemento arquitectónico básico de la ciudad jardín, es la casa unifamiliar - Cottage - con un pedazo de terreno, a modo de recordatorio de casa rural. Es rechazada la casa colectiva de pisos.

Este tipo de edificación fue utilizada en Vizcaya de manera mayoritaria, en las Cooperativas de Casas Baratas. El concepto de ciudad jardín en el País Vasco difiere del de los utópicos socialistas franceses e ingleses y de los industriales filantrópicos.

14 Archivo de Altos Hornos de Bizkaia: $n .^{\circ}$ de registro $56, n .^{\circ}$ de orden 81 
bajadora. Probablemente a Smith le resultó atractiva la propuesta, ya que en las bases del concurso se planteaba la realización de estas viviendas como modelo para las construcciones de la villa.

\subsection{Proyecto de las Calzadas de Mallona}

En 1909, mientras el Estado estudiaba el Anteproyecto de Ley que había redactado el Instituto de Reformas Sociales ${ }^{15}$, dos años antes, y que serviría de base para la Primera Ley de Casas Baratas (1911), un grupo de bilbaínos concibió el proyecto de constituir una Sociedad Anónima, cuya finalidad fuese la de construir casas baratas e higiénicas para la clase obrera.

La Sociedad Anónima de Construcciones Baratas, nombre que le dieron a la sociedad, se fundó en 1909 , con un capital de 125.000 pesetas ${ }^{16}$, distribuido en 250 acciones nominativas de 500 pesetas cada una. El proyecto, por el que se instauró dicha sociedad, era el de construir viviendas para la clase trabajadora, en régimen de alquiler, en el terreno que dicha sociedad poseía en Calzadas de Begoña, en Bilbao, frente al cementerio de Mallona, próximo a la basílica. El terreno, en ligera pendiente, guardaba las medidas higiénicas y salubres que se recomendaban en aquel momento: buena orientación y aireación, soleado durante todo el día y con vistas sobre Bilbao.

El presidente de dicha sociedad era Daniel Aresti (implicado al mismo tiempo en la construcción de Neguri), que había comprado el terreno a Luis Urrutia, en 1906, y una vez fundada la sociedad, en 1909, lo vende a dicha entidad.

Manuel Maria de Smith, a través de la Asociación de Arquitectos de Vizcaya, recibió la convocatoria al Concurso de proyectos para la edificación de viviendas obreras. Fue una circular que se envió a todos los arquitectos asociados. Por una cara estaban las condiciones del concurso con fecha del 16 de abril de 1909, firmado por el secretario de la Asociación Domingo Epalza, y en el reverso, con fecha del día siguiente, el presidente de la Asociación de Arquitectos le exhortaba a que se presentara al concurso en cuestión.

15 El Instituto de Reformas Sociales se habia fundado en 1903, en sustitución de la Comisión de Reformas Sociales que siempre tuvo un carácter benéfico.

${ }_{16}$ En los Estatutos de la Sociedad quedaba excluída la idea de lucro, al determinar que el mayor interés que podría cobrarse por las acciones fuera de un $3 \%$. 
Smith, que para entonces ya contaba con una nutrida cartera de la clientela bilbaína más selecta ${ }^{17}$, (el título de arquitecto se lo habían expedido en 1904) decide presentarse al concurso con un proyecto elaborado conjuntamente con su compañero Marcelino Arrupe ${ }^{18}$.

No se ha podido encontrar los originales ni las copias del proyecto ${ }^{19}$, pero se cuenta con abundantes borradores. Algunos muestran los bocetos de las casas que idearon, otros los cálculos económicos de los gastos de construcción y de terreno, también un borrador de la memoria y del presupuesto pormenorizado de los materiales de construcción necesarios ${ }^{20}$.

Los promotores de la construcción, a través de la convocatoria, pedian que se tuviera en cuenta varias condiciones: que fueran de planta baja y dos alturas; la máxima independencia y amplitud por vivienda; así como que se ajustaran al presupuesto económico que no debía sobrepasar las 20 pesetas de alquiler al mes por vivienda, cifra que debería servir para recuperar un $5 \%$ del precio teórico del conjunto de construcciones, siendo el precio del terreno de 70 céntimos por pie cuadrado.

Según cálculos anotados por Manuel María Smith, la medida del terreno era $62.462^{\prime} 33$ pies cuadrados, que a $0^{\prime} 70$ pesetas resultaba $43.723^{\prime} 63$ pesetas el precio total del terreno.

Por otro, Smith y Arrupe fijaron en 14 pesetas el coste del pie cuadrado de construcción y destinaron 27.000 pies cuadrados de terreno para construir, lo que ocasionaba un gasto de 378.000 pesetas, y proporcionaba una amplia zona para jardines, más de 35.000 pies cuadrados.

Con estas premisas barajaron diferentes posibilidades, cinco en total, clasificadas con las letias A, B, C, D y E. En todas ellas planteaban casitas de baja densidad, ya fueran de dos o tres alturas. Al final se decidieron

Entre ellos se encontraba Daniel Aresti, presidente de la Sociedad de Construcciones Baratas, al que habia proyectado su casa de campo en Neguri, en 1904, y una casa de alquiler, bifamiliar, con alguna caracteristica semejante a la anterior (torreta en esquina). En fechas próximas al concurso de casas Baratas de Mallona, le encargó el proyecto de una casa de vecinos de cinco alturas en Calzadas de Mallona, un taller en la calle Luchana, frente a la futura casa del propio Manuel M. ${ }^{a}$ de Smith, y en la Rivera de Deusto, una fábrica para manufacturas de la alambre. Archivo de José Maria Smith Solaun. Rollo 358, Expediente 20.

Con Marcelino de Arrupe ya había colaborado. Primero lo hizo en 1905 en la residencia de los Jesuitas de Durango, y después en 1906, cuando la Diputación de Bizkaia organizó un concurso para el retablo del Beato Valentín de Berriochoa en la iglesia de la Purisima en Elorrio, donde resultaron ganadores. PALIZA, M. Op. cit. Págs. 415, 423 y 424.

19 Según conversación mantenida con su hijo Don Juan Carlos Smith Prado (30-5-1998), es probable que entregara los originales sin hacer copia y que no se recuperaran por ser un concurso público.

${ }_{20}$ Archivo de D. José Maria Smith Solaun. Expediente 358, rollo 20. 
por casas dobles, en dos modelos distintos, seis dobles y cinco dobles con dos manos, sumando un total de 32 viviendas.

Defendieron su proyecto en base a las condiciones de la convocatoria, donde se remarcaba la importancia de la independencia y amplitud. Para dar solución al primer punto se inclinaron por la vivienda aislada, lo que resulta coherente con la trayectoria de Smith que siempre se mostró partidario de la vivienda unifamiliar para todo tipo de clase social. Aunque también reconocía la necesidad de la vivienda de pisos ${ }^{21}$ en ciertas circunstancias, cuando la construcción se levantaba en zonas industriales con mucha población, cuando existía falta de terreno o carestía del mismo...

En las Calzadas, Smith y Arrupe sólo se plantean la vivienda en casitas, aunque destinan cada altura a dos familias $y$, en base a un apunte realizado en los borradores del proyecto, es muy probable que fueran casas adosadas, con el fín de abaratar costes en favor de los metros útiles. Soluciones que, por otro lado, aplicará de manera parcial en las Casas Baratas de Altos Hornos y, que es muy probable que se inspirara en una solución de planta semejante a la de Bedford Park ${ }^{22}$.

Persiguen dar independencia a cada familia y, para que ésta sea completa, separan los accesos a cada vivienda. La planta baja tiene acceso directo por el jardín y el segundo piso a través de una escalera exterior. La escalera común es un elemento sobre el que teorizó y que parece no gustarle, como muestra en la memoria de este proyecto:

Con sólo dos alturas conseguimos evitar la odiosa escalera común, causa de grandes perjuicios de orden moral. Desde el jardín tiene cada habitación entrada independiente y además se ha optado por la escalera exterior para darle aún mayor despejo ${ }^{23}$.

Sobre las escaleras reflexionará más veces. En las grandes mansiones incluía escaleras de servicio ya que, como se deduce de lo expuesto en la cita anterior, Smith procuraba separar por medio de la escalera a las comunidades que vivian en un mismo edificio. Nueve años después de re-

"SMith IBARRA, Manuel Maria. "Urbanizaciones" en / Congreso de Estudios Vascos, Euzko Ikaskuntza. Oñate, 1918. Ver epígrafe número 7, titulado "La casa de familia y la de pisos o vecindad".

${ }_{22} \quad$ Las casas de la ciudad jardín de Bedford Park, Inglaterra, diseñadas por Norman Shaw en 1877 en el estilo Reina Ana. Estilo este que él mismo, junto a Wed y Nesfield habían creado a partir de de las tradiciones domésticas inglesas y holandesas diseñó cinco prototipos de casas dobles, sencillas y adosadas. Bedford Park alcanzó las quinientas casas, fue un lugar muy reconocido ya que lo eligieron artistas e intelectuales para residencia

23 Archivo de D. José María Smith Solaun. Rollo 358, Expediente 20. 
dactar dicha memoria criticará abiertamente las escaleras en el I Congreso de Estudios Vascos ${ }^{24}$.

En cuanto a la amplitud de las viviendas, segundo punto mencionado en la convocatoria del concurso de las Calzadas, es el apartado en el que más se extiende la memoria. Considera el espacio como algo imprescindible para la calidad de vida del trabajador, destina un dormitorio para el matrimonio, otro para los hijos varones y el otro para las chicas, quedando un tercer hueco que se señala como posible sala, comedor, o dormitorio según los gustos. Precisamente se apoya en el tamaño de la vivienda para defender su propuesta:

...y creyendo que no hacemos casas para pordioseros sino para trabajadores, nos hemos esforzado en dar la amplitud relativa que se ve en nuestros planos. Además la poca altura de las casas contribuye a dar aún mayor ambiente y obedece a una ventaja de gran peso para nosotros, como es seguramente para esa Sociedad, la facilidad del agua para subir al piso ${ }^{25}$.

Smith y Arrupe proyectan, en base al presupuesto económico, unas casas bastante amplias, con unas medidas de diez metros por siete de planta, repartidos en tres dormitorios, sala, cocina-comedor, retrete, despensa y terraza. Optan por dos distribuciones (fig. 1), siendo la cocina-comedor, pieza principal en ambos. De ella parten el resto de huecos, cumpliendo así la función de pieza central, recibidor, distribuidor, hogar y cuarto de estar. En esta distribución, que también utilizará Smith en las casas para obreros de la Fábrica Rica Hermanos de Zaramillo, hay cierta reminiscencia del estilo Reina Ana o del Shingle Style americano.

Al haberse perdido los documentos originales, desconocemos cuales fueron los proyectos de los alzados, pero resulta lógico colegir, en base a lo que construyó posteriormente, que utilizase el estilo inglés basándose en el repertorio Old English o en el Reina Ana, estilos que utilizaba en ese momento para las mansiones de Neguri, y que posteriormente utilizará en la casa de Altos Hornos, en Barakaldo.

De cualquier modo el proyecto estudiado no pudo llevarse a cabo ya que fue desestimado en favor del de Enrique Epalza Chanfroau, quien se decidió por unas viviendas en bloques, de planta baja y dos pisos, con un espacio interior más reducido: tres huecos, cocina, y retrete. Esta solución resultaba más rentable económicamente a la Sociedad

${ }^{24}$ Smith lbarra, Manuel María. Op. cit. pág. 7.

25 Archivo de D. José María Smith Solaun. Rollo 358, Expediente 20, Memoria. 


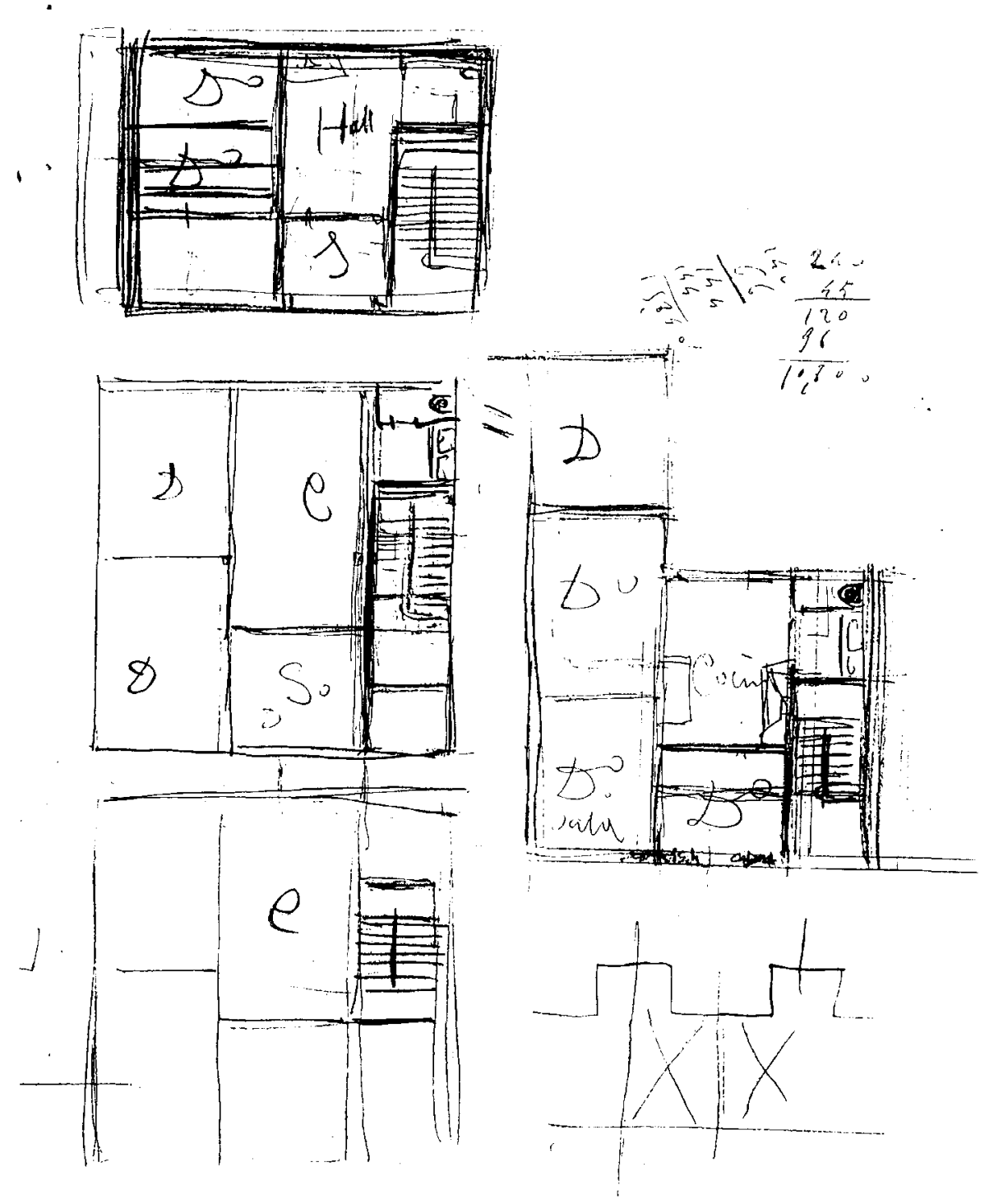

Figura 1. Plantas de las viviendas del concurso de Calzadas de Begoña. Año 1909. 
Constructora y se podía habitar por mayor número de familias. Además el que dispusiera de menos habitaciones fue un elemento determinante ya que, con la reducción de espacio por vivienda, la Sociedad evitaba el subarriendo.

Dieciséis casitas de dos alturas con entradas independientes para cada familia, rodeadas de jardín,... son datos que extraemos de los abundantes borradores que se conservan y que junto a las especificaciones de la desestimación del proyecto por parte de la Sociedad convocante, donde se señala que guardarán su propuesta para unos terrenos mas baratos en las afueras (fig. 2), nos llevan a pensar que Smith habia planteado una urbanización en la línea de las garden cities semejante a la que años después realiza para Altos Hornos de Vizcaya.
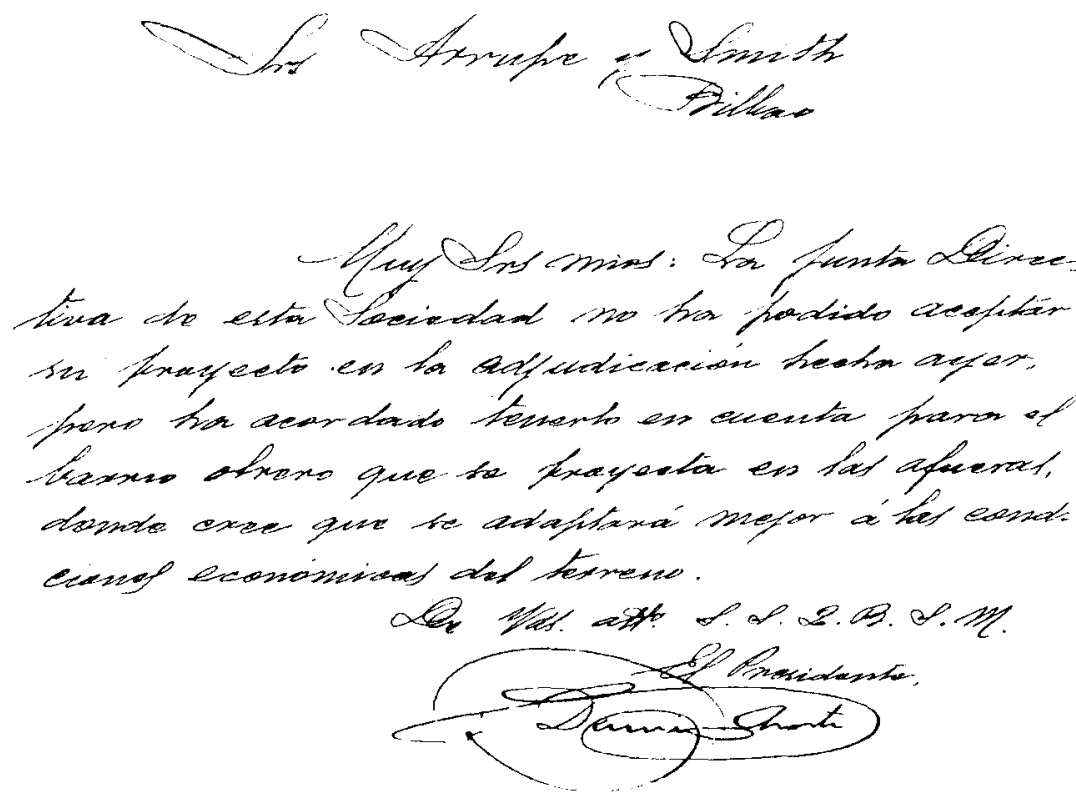

Figura 2. Notificación a Arrupe y Smith desestimando el proyecto presentado al concurso de Calzadas de Begoña. Año 1909. 
En este sentido, es interesante señalar que Manuel María Smith habia acudido, tan solo dos años antes de redactar este proyecto, en 1907, al Congreso Internacional de Higiene y Demografía que se celebró en Berlín, en el que se abogó por la construcción de ciudades jardín.

\subsection{Las viviendas de Altos Hornos de Vizcaya de San Vicente, en Barakaldo}

Manuel María Smith tuvo a lo largo de su vida una intensa relación profesional con la empresa Altos Hornos de Vizcaya.

La primera obra que le encargó AHV, en 1908, fue con motivo de la Exposición Hispano- Francesa de Zaragoza, donde la empresa presentaba una instalación industrial y Smith realizó el tratamiento artístico. Dos años después le encarga un sanatorio para los empleados de la empresa, en el que experimentó con el Sezzesionismo. En 1911 proyecta un nuevo pabellón de especialidades para este Sanatorio, que sin embargo no se llegó a construir. El mismo año lleva a cabo dos reformas, una en las antiguas oficinas de Altos Hornos de Vizcaya ${ }^{26}$, y la otra en la capilla del Carmen en el Desierto de Barakaldo ${ }^{27}$.

Para la misma empresa, pero en el municipio de Sestao, proyecta y presupuesta las escuelas para la Sociedad Altos Hornos de Vizcaya. Finalmente no lleva a cabo esta obra, pero la empresa delega en él la evaluación y selección de los nuevos proyectos presentados por otros arquitectos. De esta manera Smith propone al Sr. D. José Lastra ${ }^{28}$.

Este dato nos hace pensar que la relación de la empresa con el arquitecto, era estrecha y que no se reducía exclusivamente a la de cliente-arquitecto. De cualquier modo la lista de trabajos realizados para Altos Hornos de Vizcaya es aún más extensa: proyecto de Sanatorio para los Obreros de Altos Hornos en Sestao, nuevas oficinas de Altos Hornos en Barakaldo, construidas en 1946, y las Casas Baratas, motivo de nuestro estudio.

A finales de 1914, ante la carencia que sufría Barakaldo de viviendas para los obreros, se crea la Sociedad de Casas Baratas de Barakaldo y

${ }^{26}$ A partir de esta fecha, la relación con los directivos de la empresa se hace más estrecha, sobretodo con Victor Chávarri, para el hace el palacio Artaza, en Leioa, obra cumbre de Smith, y reforma el palacio perteneciente a su esposa en Zugazarte, Getxo.

${ }_{27}$ Archivo de D. José Maria Smith Solaun: Carpeta E- 3. Expediente. 23.

$28 \quad$ Ibidem. Folio 6 y siguientes. 
Sestao, con participación directa de los directivos de Altos Hornos ${ }^{29}$. Se decide realizar una barriada de casas para los operarios, en régimen de alquiler, escogiéndose como lugar de su ubicación un terreno plano en el barrio de San Vicente, cercano a la iglesia del mismo nombre.

Se le encarga el diseño de la obra a Smith, que firma el primer proyecto el 10 de noviembre de $1916{ }^{30}$. Elige un tipo de construcción cercano al de la Ciudad Jardín. Dado lo similar del planteamiento, parece que intenta llevar a la práctica lo que en 1909 había proyectado para el concurso de Calzadas. Diseña casas adosadas (fig. 3) de diferente tamaño y forma: ocho unifamiliares, de planta baja y primer piso, y tres casas con dos manos y dos alturas cada una, lo que da doce viviendas. En total suponen un proyecto de 20 viviendas, cuya solución recuerda enormemente a la proyectada en las Calzadas y nos vuelve a remitir a Inglaterra con Bedford Park ${ }^{31}$.

El terreno que ocupan es de unos 2.100 metros cuadrados, rodeado de campo, bien aireado y soleado, actualmente tienen un hermoso parque municipal frente a las fachadas principales, aunque por detrás el barrio ha crecido en exagerada densidad y altura.

El precio de la construcción según el cálculo realizado por Alfredo Acebal ${ }^{32}$, arquitecto municipal de Barakaldo, fue de 90.000 pesetas.

El permiso de habitabilidad para el primer grupo de viviendas del barrio de San Vicente ${ }^{33}$ fue solicitado el 20 de febrero de 1918 por Smith, en nombre de la Sociedad de Casas Baratas. El alcalde de Barakaldo Juan de Garay pide la evaluación de la finca para el cobro de los respectivos impuestos así como que pasen los técnicos para comprobar si se ha acometido el alcantarillado a la red general, y el arquitecto municipal, Alfredo Acebal, concede la habitabilidad previa descripción del número de habitaciones de que consta la construcción y del precio para el cálculo posterior del impuesto de construcción y alcantarillado:

.. ocho son sencillas de piso bajo y principal, dedicadas a una sola vivienda y tres dobles, de piso bajo y principal, con un total de cuatro habita-

29 Esta sociedad, según sus estatutos, artículo $2 .^{\circ}$ tenia por objetivo la adquisición y urbanización de terrenos convenientes para la construcción de barrios o viviendas obreras. Archivo de Altos Hornos de Bizkaia: número de registro 56, número de orden 81.

30 Archivo del Ayuntamiento de Barakaldo. Sección Fomento, Carpeta 159 - 1

${ }^{31}$ Donde el promotor de la urbanización Jonatan T. Carr en 1876, solicitó al arquitecto Edward William Godwin (1833-1886) varios prototipos de casas para alternar y repetir.

${ }^{32}$ Archivo Municipal de Barakaldo. Sección Fomento, letra B, Carpeta n. ${ }^{\circ} 45$. Año 1918, Negociado 11.

${ }_{33}$ Ibidem, Carpeta 158, n. ${ }^{\circ} 45$, Año 1918, Negociado 11. 
ciones cada una [...] El coste aproximado a mi juicio es de noventa mil pesetas y todas ellas acometen al alcantarillado general ${ }^{34}$.

Manuel María de Smith distribuyó sobre el terreno una hilera de casas, de diferentes tamaños. Las más pequeñas tenían 56 metros cuadrados en una sola planta, otras tenían 84 metros, divididos en dos plantas, y un tercer tipo contaba con 96 metros cuadrados, también en dos plantas. Todas contaban con cocina, retrete, tres habitaciones y las grandes con cuatro habitaciones más despensa. Se da un caso especial, el de la casa situada en la esquina, que cuenta con cinco habitaciones y dos retretes, uno en cada piso.

Cada casa poseía un pequeño jardín en la parte delantera y en la trasera un patio, al que se accedía desde la cocina.

Las fachadas muestran el programa de los elementos del Old English. Juega con la asimetría, provocada por el retranqueo del segundo cuerpo de casas, por los hastiales de remates triangulares de diferente tamaño, altura y distinta decoración, a base de entramados ficticios tanto de líneas

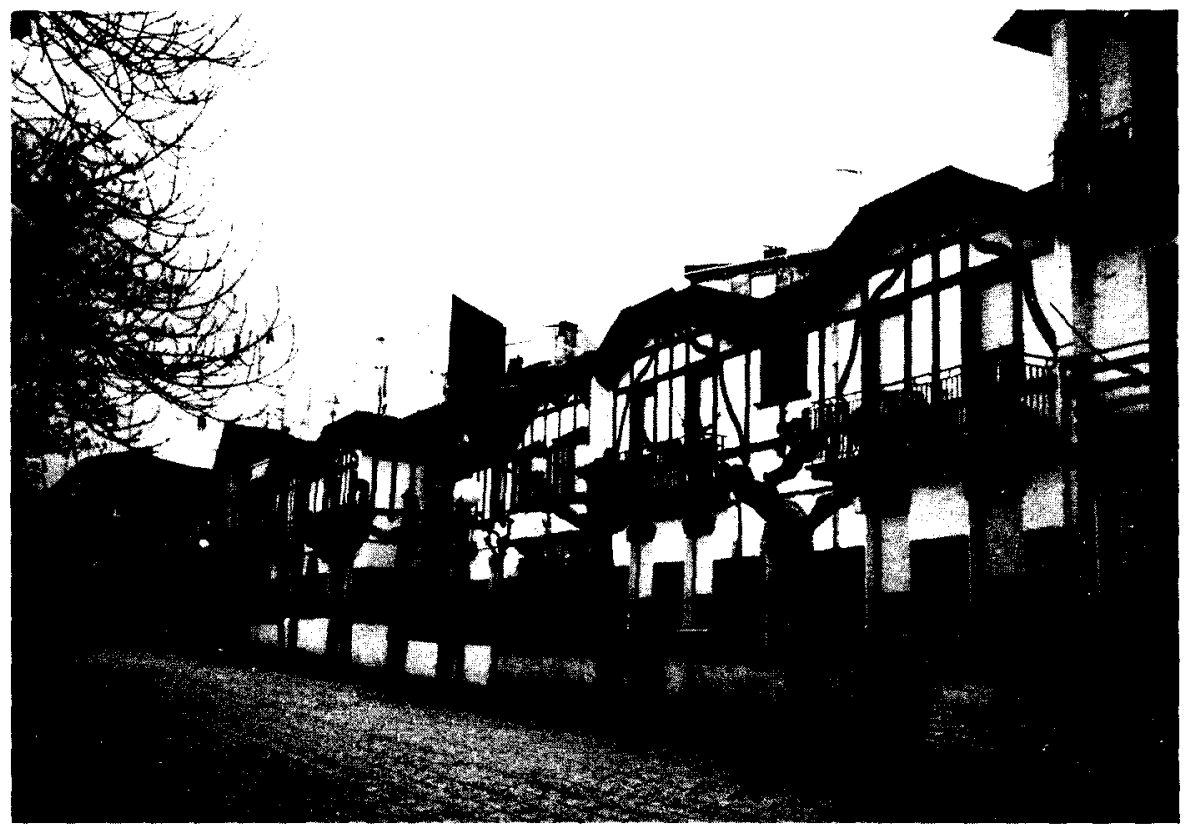

Figura 3. Casas Baratas de Altos Hornos de Vizcaya, en San Vicente, Barakaldo. Año 1916. Arquitecto D. Manuel Maria de Smith Ibarra.

34 Ibidem, Folio 2. 
rectas como curvas (fig. 4). Las fachadas son ricas en balcones con antepechos de madera, las ventanas de diferentes tamaños algunas decoradas con ladrillo visto al igual que en las puertas de arco de medio punto. También incluye oriel windows, y esquinas rematadas en torres (fig. 5) que ayudan a darle un carácter más exquisito al conjunto. Como nota popular pone cortafuegos decorativos, separando los distintos cuerpos y en las torres; también incluye otra nota decorativa muy utilizada en la arquitectura de tendencia neovasca, la hornacina, para incluir una imagen sagrada (fig. 6).

Una vez terminadas estas 20 viviendas, añade cuatro casas más, perpendicularmente a la esquina, formando una $L{ }^{35}$ Smith pidió la habitabilidad de este bloque el 2 de junio de $1920{ }^{36}$, lo que nos permite deducir que prácticamente las había empezado a la vez que la segunda fase que proyecta en 1918. En un principio este proyecto de segunda fase era de

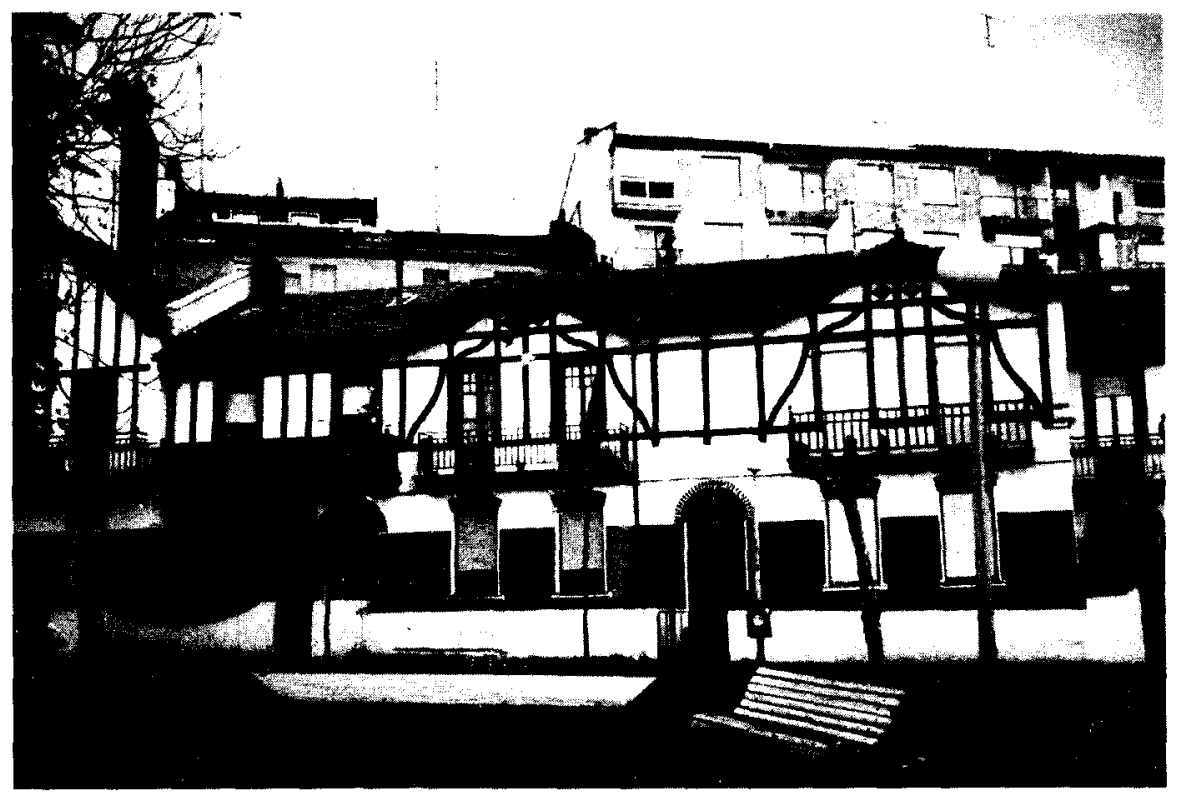

Figura 4. Casas Baratas de Altos Hornos de Vizcaya, en San Vicente, Barakaldo. Año 1916. Arquitecto D. Manuel Maria de Smith Ibarra.

\footnotetext{
${ }^{35}$ Ese terreno pertenecia a la iglesia que, dos años después, accedió a cambiarlo por otros que le interesaban y que pertenecian al ayuntamiento. La Sociedad de Casas Baratas debió gestionar con el ayuntamiento el cambio de terrenos. Archivo Municipal de Barakaldo, Bizkaia, Sección Fomento, Año 1918 Letra B.9.1. Carpeta 158, n. ${ }^{\circ} 45$, Negociado 11.

${ }^{36}$ Archivo Municipal de Barakaldo, Bizkaia, Sección Fomento, Año 1920 Carpeta 160, n. 55.
} 

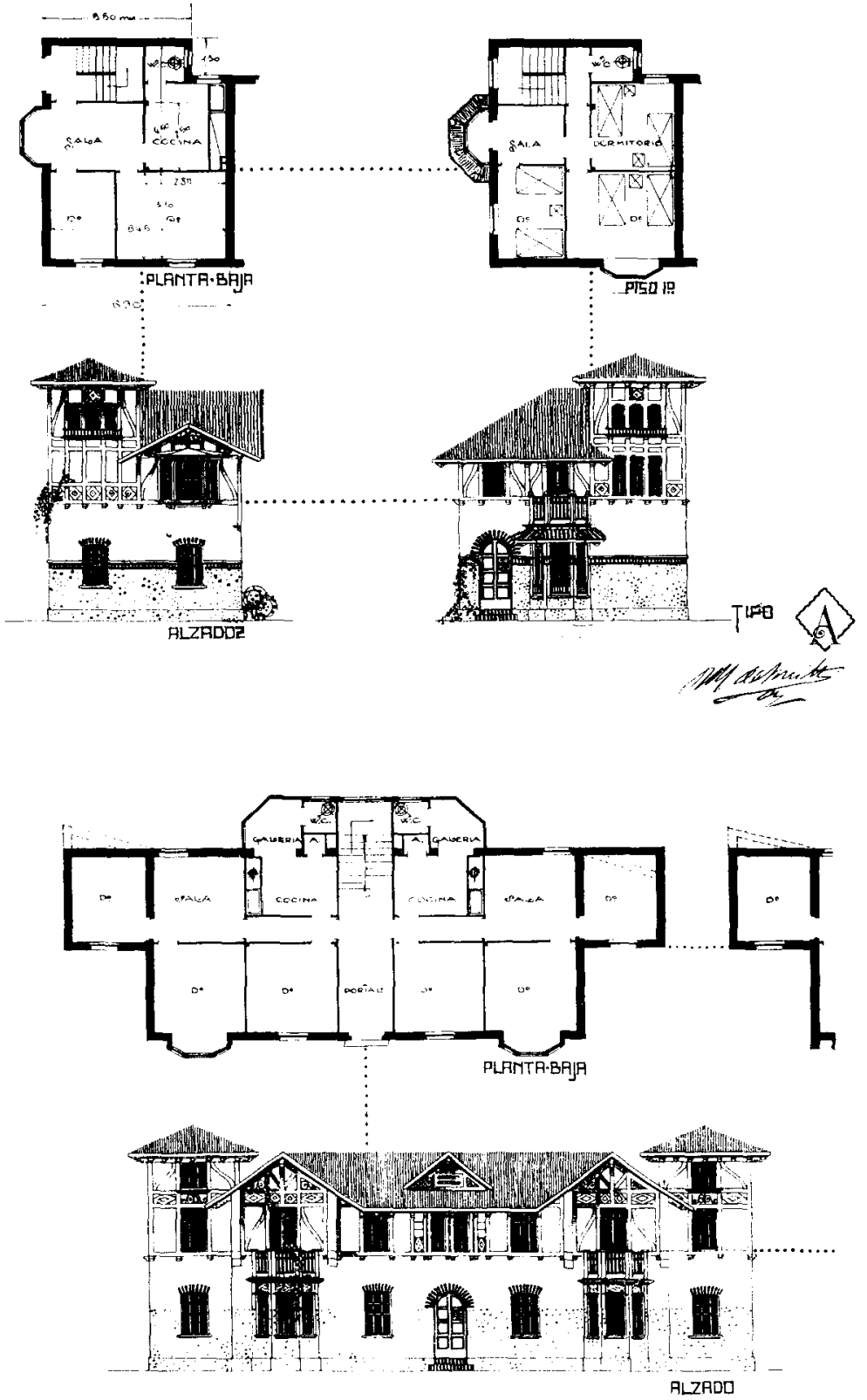

Figura 5. Anteproyectos de diferentes esquinas, rematadas en torre. Casas Baratas de Altos Hornos de Vizcaya, en San Vicente, Barakaldo. Año 1918. Arquitecto D. Manuel María de Smith lbarra. 


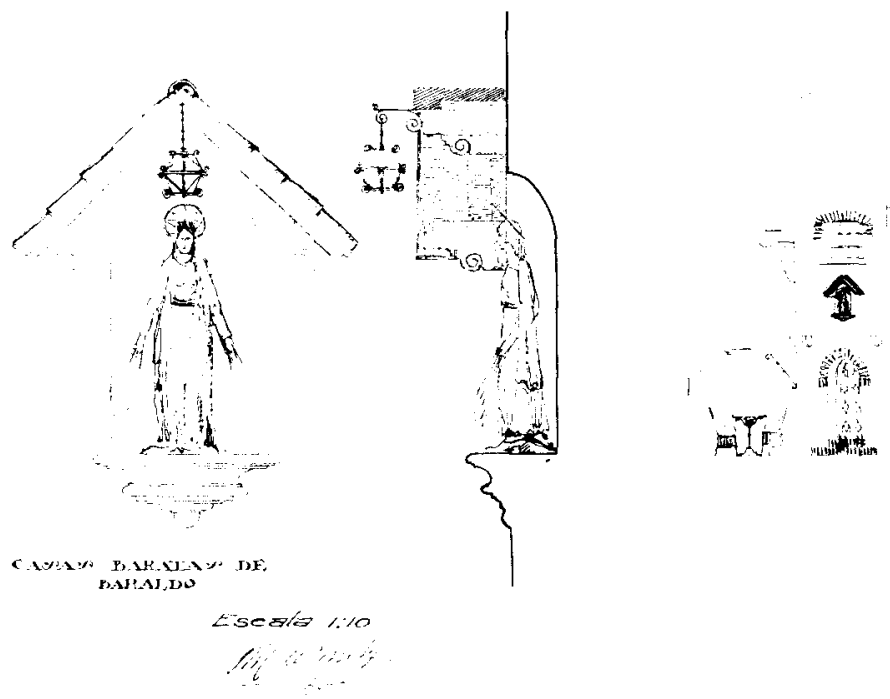

Figura 6. Dibujo a plumilla de una imagen para hornacina en las Casas Baratas de Altos Hornos de Vizcaya, en San Vicente, Barakaldo. Año 1918. Arquitecto D. Manuel María de Smith Ibarra.

tres manzanas y otra hilera donde, a parte de comercios, se haría un edificio exento para baños y gimnasio (fig. 7). Finalmente sólo se realizó una manzana en línea con lo ya construido.

Aunque Smith proyectó más de diez tipos de casas diferentes, tanto para la primera fase como para las posteriores, tuvo que reducir y simplificar el programa arquitectónico y decorativo. En la segunda fase se vio obligado a incluir pisos en dos y tres alturas. El resultado perdió en estética respecto al primero pero salió más rentable económicamente y acogió a mayor número de personas.

\subsection{Casas de obreros para la Sociedad Rica Hermanos, en Zaramillo}

El mismo año que proyecta las Casas Baratas para Altos Hornos, lo hace para la Sociedad Rica Hermanos ${ }^{37}$. Esta empresa se dedicaba a la

${ }_{37}$ Dicha empresa había sido denunciada varias veces, unas por falta de higiene y por no tener suficiente luz y ventilación, otras por contratar a mujeres y niños menores de 14 años. Las denuncias 

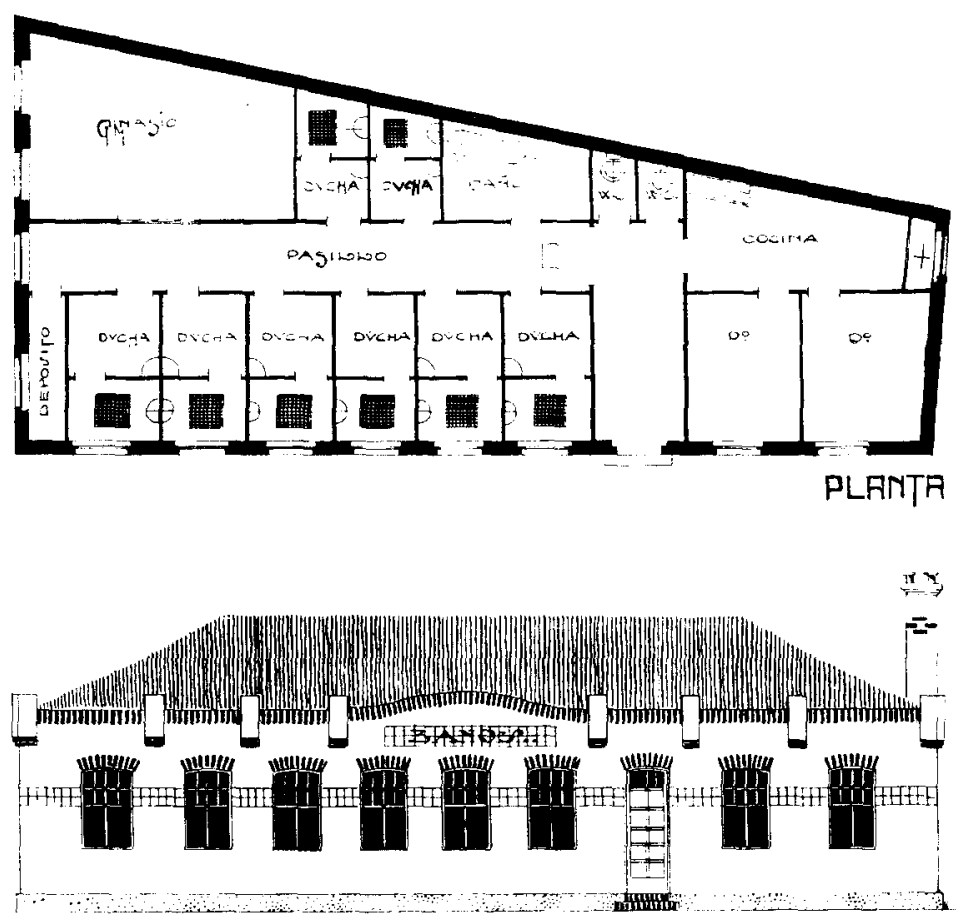

FLZRDO

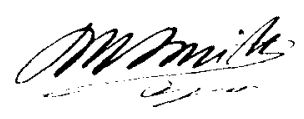

Figura 7. Anteproyecto para baños y gimnasio en las Casas Baratas de Altos Hornos de Vizcaya, en San Vicente, Barakaldo.

Año 1918. Arquitecto D. Manuel Maria de Smith Ibarra.

se cursaron por la Junta Local de Reformas Sociales de Barakaldo el 10 de febrero de 1912, el 22 de febrero de 1913, el 30 de marzo de 1915. El 29 de diciembre de 1915 la Junta mencionada recibe una carta de Rica Hermanos, diciendo que contratan a menores porque al tener las fábricas alejadas de los municipios no acuden los obreros y tienen incluso máquinas sin funcionar por falta de personal. Por lo que han dispuesto construir habitaciones económicas con el objeto de crear un núcleo de población adjunto al establecimiento industrial. Archivo Municipal de Barakaldo. Actas de Reformas Sociales. Carpeta $462, \mathrm{n} .{ }^{\circ} 8$, año 1912 y siguientes, págs. $3,37,39,95$, y 119 a 1123. 
producción de derivados del yute. Estaba situada en un medio totalmente rural, combinando autonomía económica y energética, con fuertes lazos de dependencia entre el empresario paternalista y el trabajador, integrando trabajo y vida privada, mano de obra barata y poco conflictiva captada del medio rural.

Con fecha del nueve de marzo de 1916, Smith presenta en el ayuntamiento Baracaldés el proyecto de casas de alquiler para los señores Rica Hermanos en Zaramillo, en el Barrio Irauregui, de Barakaldo ${ }^{38}$. Se trata de tres edificios situados en la carretera de Bilbao a Balmaseda. Cada uno consta de planta baja, dos pisos y buhardilla en mansarda (fig. 8).

L.as viviendas son pequeñas, con aproximadamente 42 metros cuadrados, y distribuidas en tres dormitorio, cocina y retrete, menos en la planta baja que hay un piso aún más pequeño, de sólo dos dormitorios. Todas las habitaciones se abren a la cocina, en una solución idéntica a la utilizada en el proyecto que presentó al concurso de las Calzadas y que señala indefectiblemente a los estilos Reina Ana o Shingle Style americano. Con esta solución del interior, se adelanta al planteamiento de vivienda minima que una década después será tema constante en el planteamiento de vivienda.

Las fachadas, muy sencillas, sin ningún tipo de decoración exceptuando la línea de imposta y las ventanas que se apoyan en ladrillos vistos. No existen ni miradores ni balcones. La bajo cubierta es alta, para poder habitarla. Se aleja de la propuesta que él había empleado para el concurso de Casas Baratas de Calzadas de Mallona (hay que tener en cuenta que este es un encargo muy específico por parte de la empresa) y se acerca a la solución que dio el ganador, Enrique Epalza, de fachadas planas con apenas más decoración que la línea de imposta, y con interiores de reducido tamaño.

Cuatro meses después, con fecha del dieciocho de julio de 1916 presenta el proyecto de un nuevo pabellón ${ }^{39}$ para Rica Hermanos, destinado a talleres y un edificio destinado a oficinas, guardarropa y vivienda del director. En la sesión celebrada en el Ayuntamiento el 18 de agosto de 1916, se lee el informe para la construcción de lo solicitado.

Smith elige para este edifico una fachada más trabajada que las anteriores. La tipologia que utiliza es de casa-torre, y proyecta un edificio cúbico de planta baja y dos pisos. En la baja sitúa el guardarropa, lavabos, W.C, y portería, en el primer piso oficinas y el segundo lo habilita como vivienda

38 Archivo Municipal de Barakaldo, Bizkaia, Sección Fomento, Carpeta 156, N. 25.

39 Ibidem, N. ${ }^{\circ} 26$. 


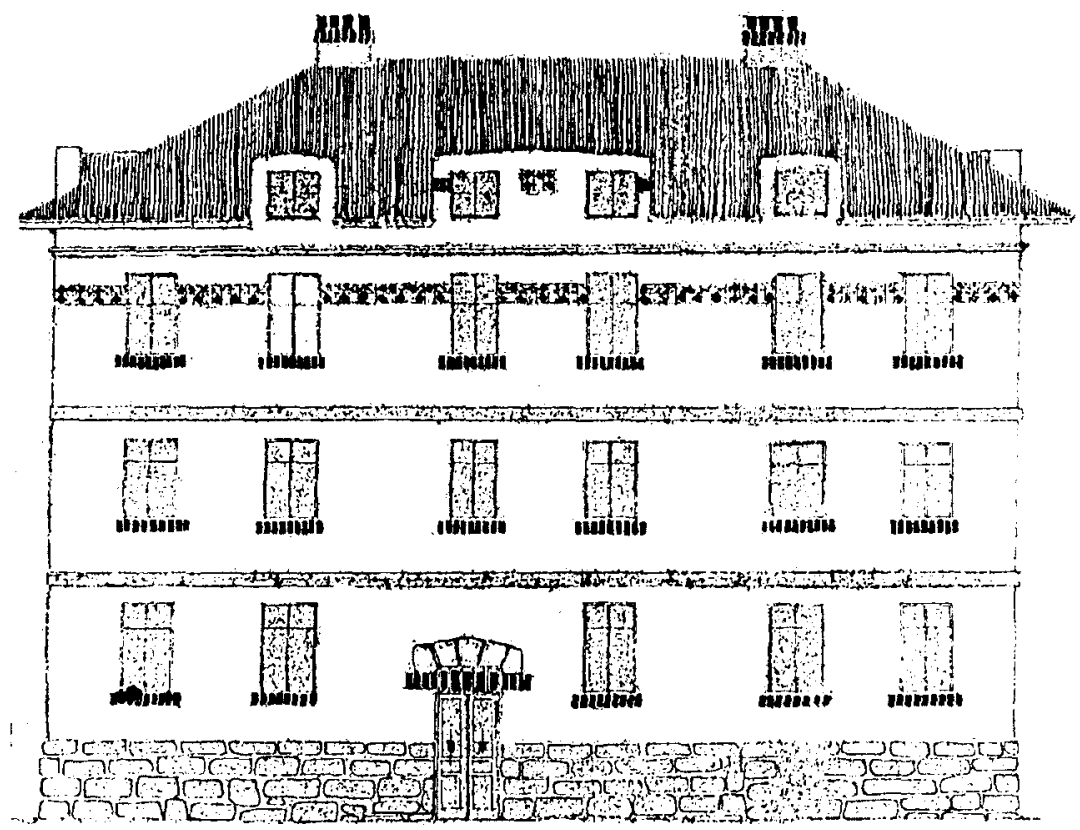

FTRE, YTE

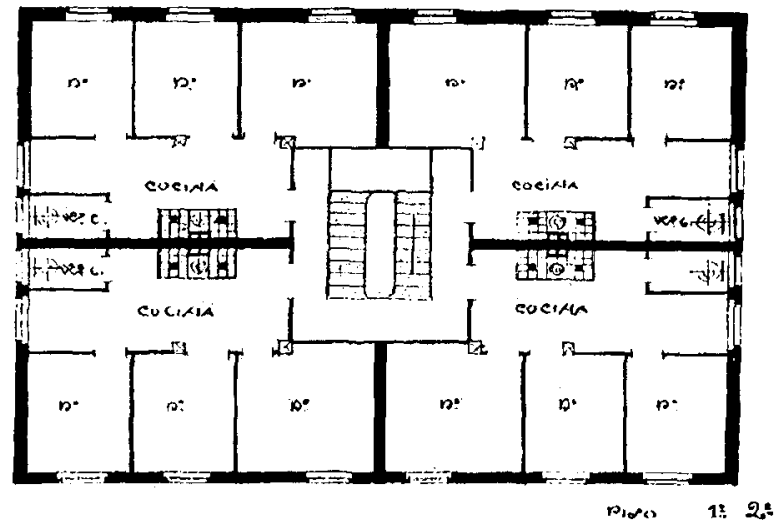

Figura 8. Plantas y alzados de las casas para obreros de la fábrica Rica Hermanos, en Irauregui, Barakaldo. Año 1916. Arquitecto D. Manuel Maria de Smith Ibarra. 
para el director. En esta vivienda destacamos el baño, con hermosa bañera e inodoro incluido en el mismo recinto, existiendo otro hueco más donde presenta exclusivamente un inodoro. Es una vivienda de cuatro dormitorios, comedor, sala, despensa, cocina, hermoso hall y dos solanas. La fachada pensada en estilo neovasco recuerda a las casas-torres con cubierta a cuatro vertientes y ladrillo visto en los paramentos del último piso, línea de imposta de ladrillo en los vanos del segundo piso, dinteles monolíticos apoyados en ménsulas característica de las casas torre. Desde luego esta vivienda dista mucho en tamaño, distribución y calidad con las anteriores.

\subsection{Casas Baratas de Beurco, en Barakaldo}

El 17 de enero de 1919, de nuevo para Altos Hornos, Smith solicita permiso ${ }^{40}$ en nombre de la Sociedad de Casas Baratas para ejecutar los trabajos de edificación de las casas que desea construir dicha sociedad en el barrio de Beurco, en el municipio de Barakaldo. Los permisos correspondientes se cursan el 27 y el 30 del mismo mes. No hay más documentación al respecto, pero las obras no las realizó Smith. Tres años después es Manuel Camerón quien comienza las obras, para hacerse cargo a continuación Santos Zunzunegui, arquitecto que opta por casas unifamiliares adosadas.

Por lo tanto, en lo que respecta a Smith, de esta barriada sólo podemos decir que existió un proyecto, muy probablemente de casas de baja densidad, en la línea de las que ya había construído para la misma Sociedad y de las que él era partidario. El encargo lo recoge después Santos Zunzunegui, arquitecto que realizó en Bizkaia otros grupos de Casas Baratas, en régimen de cooperativa, siguiendo las tipologías inglesas: casas individuales con jardín y patio trasero, con tres o cuatro dormitorios, sala, cocina y retrete. Las fachadas no están tan trabajadas como las de Smith, son más monótonas, reiterativas y sencillas a pesar de que también incorpora los falsos entramados y miradores poligonales.

\subsection{Concurso de proyectos de Solocoeche}

Por último, Smith se presenta al concurso que convoca la Junta de Viviendas Municipales del Ayuntamiento de Bilbao a finales de 1931. El

40 Ibidem, Carpeta 159-1-1. 


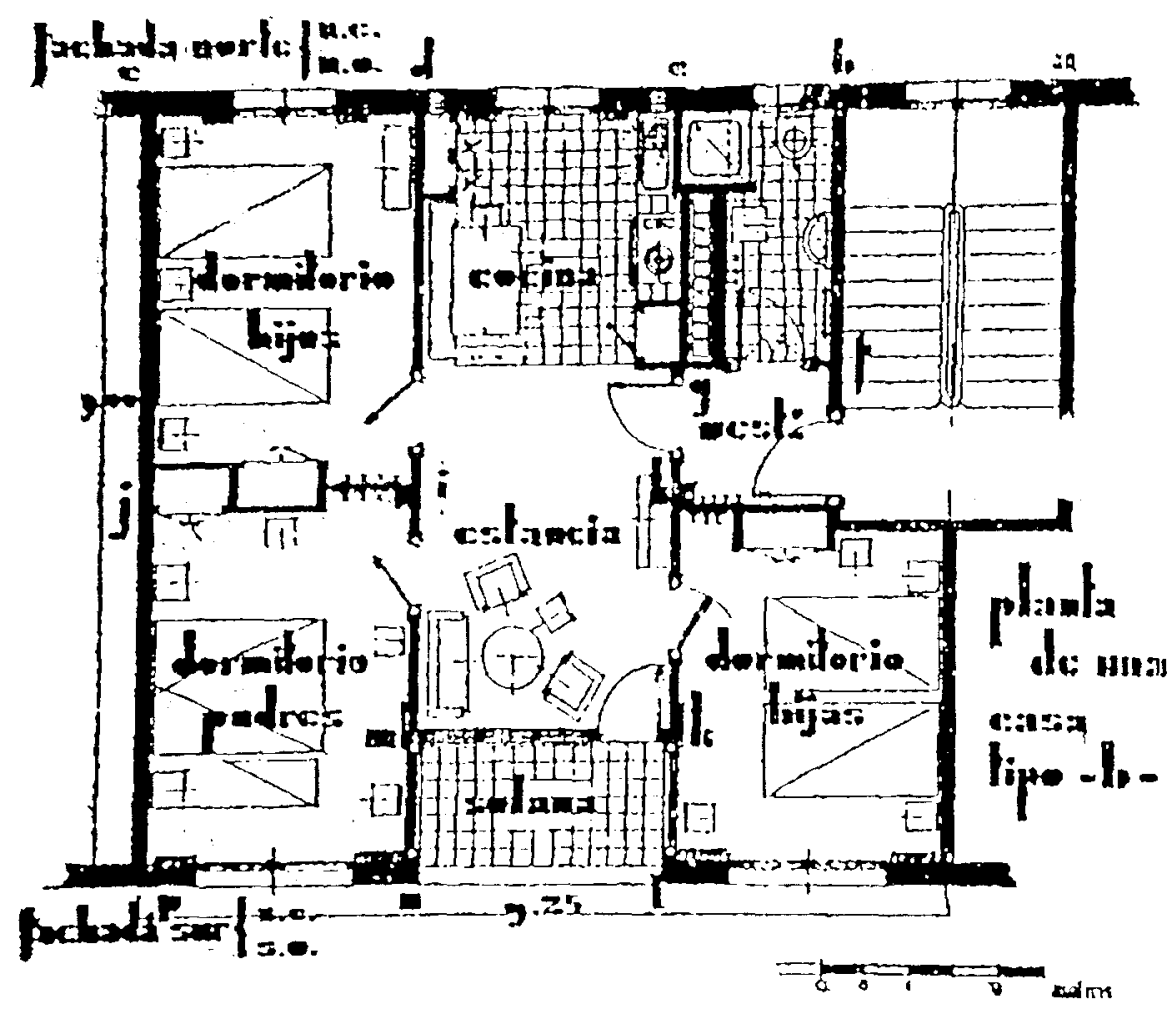

Figura 9. Planta de la vivienda del proyecto ganador para el concurso de Solocoeche, Bilbao. Año 1931. Arquitecto D. Emiliano Amann Amann.

trabajo ahora, en tiempo de crisis económica, no era tan abundante y aunque este probablemente no fuera el motivo, ya que no le faltó trabajo, la convocatoria planteaba un tipo de vivienda totalmente alejada de la tipología que habia venido realizando. En realidad era contraria a las casas unifamiliares que se habian estado realizando alentadas por las Casas Baratas. El Ayuntamiento atrajo a un gran número de arquitectos, con el atractivo de que el proyecto premiado sirviera como ejemplo para un plan de construcciones de este género que las administraciones apoyarian.

Smith supo adaptarse a los cambios que se estaban produciendo, la crisis y el estilo racionalista empezaron a ir parejos. Dejó a un lado todo su anterior repertorio artístico que tantas satisfacciones le había dado, y se centró en las demandas sociales del momento. 
Smith realizó un proyecto de bloques de pisos en $U$ ocupando el espacio central un cuerpo interior con la misma forma de $U$ donde ubicaba la zona comercial. Las viviendas costaban de tres dormitorios, cocina y retrete, que se habrian a un pasillo longitudinal.

Calixto Emiliano Amann recibe el primer premio por una solución que tiene como pieza central la cocina (fig. 9) y que curiosamente Smith habia venido proyectando desde 1909 en Calzadas, y repetido en Zaramillo. 
